\title{
Structure-guided mutational analysis of T4 RNA ligase 1
}

\author{
LI KAI WANG, ${ }^{1}$ BEATE SCHWER, ${ }^{2}$ and STEWART SHUMAN ${ }^{1}$ \\ ${ }^{1}$ Molecular Biology Program, Sloan-Kettering Institute, New York, New York 10021, USA \\ ${ }^{2}$ Immunology and Microbiology Department, Weill Medical College of Cornell University, New York, New York 10021, USA
}

\begin{abstract}
T4 RNA ligase 1 (Rnl1) is a tRNA repair enzyme that circumvents an RNA-damaging host antiviral response. Whereas the three-step reaction scheme of Rnl1 is well established, the structural basis for catalysis has only recently been appreciated as mutational and crystallographic approaches have converged. Here we performed a structure-guided alanine scan of nine conserved residues, including side chains that either contact the ATP substrate via adenine (Leu179, Val230), the 2'-OH (Glu159), or the $\gamma$ phosphate (Tyr37) or coordinate divalent metal ions at the ATP $\alpha$ phosphate (Glu159, Tyr246) or $\beta$ phosphate (Asp272, Asp273). We thereby identified Glu159 and Tyr246 as essential for RNA sealing activity in vitro and for tRNA repair in vivo. Structure-activity relationships at Glu159 and Tyr246 were clarified by conservative substitutions. Eliminating the phosphatebinding Tyr37, and the magnesium-binding Asp272 and Asp273 side chains had little impact on sealing activity in vitro or in vivo, signifying that not all atomic interactions in the active site are critical for function. Analysis of mutational effects on individual steps of the ligation pathway underscored how different functional groups come into play during the ligase-adenylylation reaction versus the subsequent steps of RNA-adenylylation and phosphodiester formation. Moreover, the requirements for sealing exogenous preformed RNA-adenylate are more stringent than are those for sealing the RNA-adenylate intermediate formed in situ during ligation of a $5^{\prime}-\mathrm{PO}_{4}$ RNA.
\end{abstract}

Keywords: RNA repair; polynucleotide ligase; adenylyltransferase; tRNA breakage

\section{INTRODUCTION}

RNA ligases join $3^{\prime}-\mathrm{OH}$ and $5^{\prime}-\mathrm{PO}_{4}$ RNA termini via a series of three nucleotidyl transfer steps: (1) RNA ligase reacts with ATP to form a covalent ligase-(lysyl-N)-AMP intermediate plus pyrophosphate; (2) AMP is transferred from ligase-adenylate to the $5^{\prime}-\mathrm{PO}_{4}$ RNA end to form an RNAadenylate intermediate (AppRNA); and (3) ligase catalyzes attack by an RNA $3^{\prime}-\mathrm{OH}$ on the RNA-adenylate to seal the two ends via a phosphodiester bond and release AMP (Cranston et al. 1974; Sugino et al. 1977; Uhlenbeck and Gumport 1982). Bacteriophage T4 RNA ligase 1 (Rnl1), discovered by Hurwitz and colleagues (Silber et al. 1972), is the founding member of the RNA ligase enzyme family. Rnl1 exemplifies a distinct subgroup of RNA ligases with a narrow phylogenetic distribution and signature structural elements that are essential for ligase activity (Wang et al. 2003). The Rnl1-like enzymes include homologs encoded by other bacteriophages (Fig. 1), the tRNA ligases of fungi and plants,

Reprint requests to: Stewart Shuman, Molecular Biology Program, Sloan-Kettering Institute, New York, NY 10021; e-mail: s-shuman@ ski.mskcc.org; fax: (212) 717-3623.

Article published online ahead of print. Article and publication date are at http://www.rnajournal.org/cgi/doi/10.1261/rna.271706. and an RNA ligase encoded by the Autographa californica baculovirus (Martins and Shuman 2004; Blondal et al. 2005; Englert and Beier 2005; Wang and Shuman 2005; Wang et al. 2006). T4 Rnl1 and yeast tRNA ligase are functionally and structurally homologous proteins dedicated to the repair of programmed tRNA breaks in vivo (Amitsur et al. 1987; Phizicky et al. 1992; Schwer et al. 2004).

The Rnl1 family is distinct from the Rnl2 family, which is exemplified by bacteriophage T4 Rnl2 and trypanosome RNA-editing ligases (McManus et al. 2001; Schnaufer et al. 2001; Ho and Shuman 2002). The Rnl2-type enzymes comprise a branch of the covalent nucleotidyltransferase enzyme superfamily, composed of DNA ligases, RNA ligases, and mRNA capping enzymes, all of which catalyze the nucleotidylation of polynucleotide $5^{\prime}$ ends via a covalent enzyme-(lysyl-N $\zeta$ )-NMP intermediate (Shuman and Lima 2004). The typical nucleotide-binding pocket of covalent nucleotidyltransferases is composed of six peptide motifs (I, Ia, III, IIIa, IV, and V) (shaded in Fig. 1) that contribute essential constituents of the active site. Knowledge of the structural basis for catalysis and substrate recognition by RNA ligases has emerged from studies of T4 Rnl2 entailing extensive mutational analyses and nucleic acid substrate modifications (Yin et al. 2003; Nandakumar et al. 2004; 


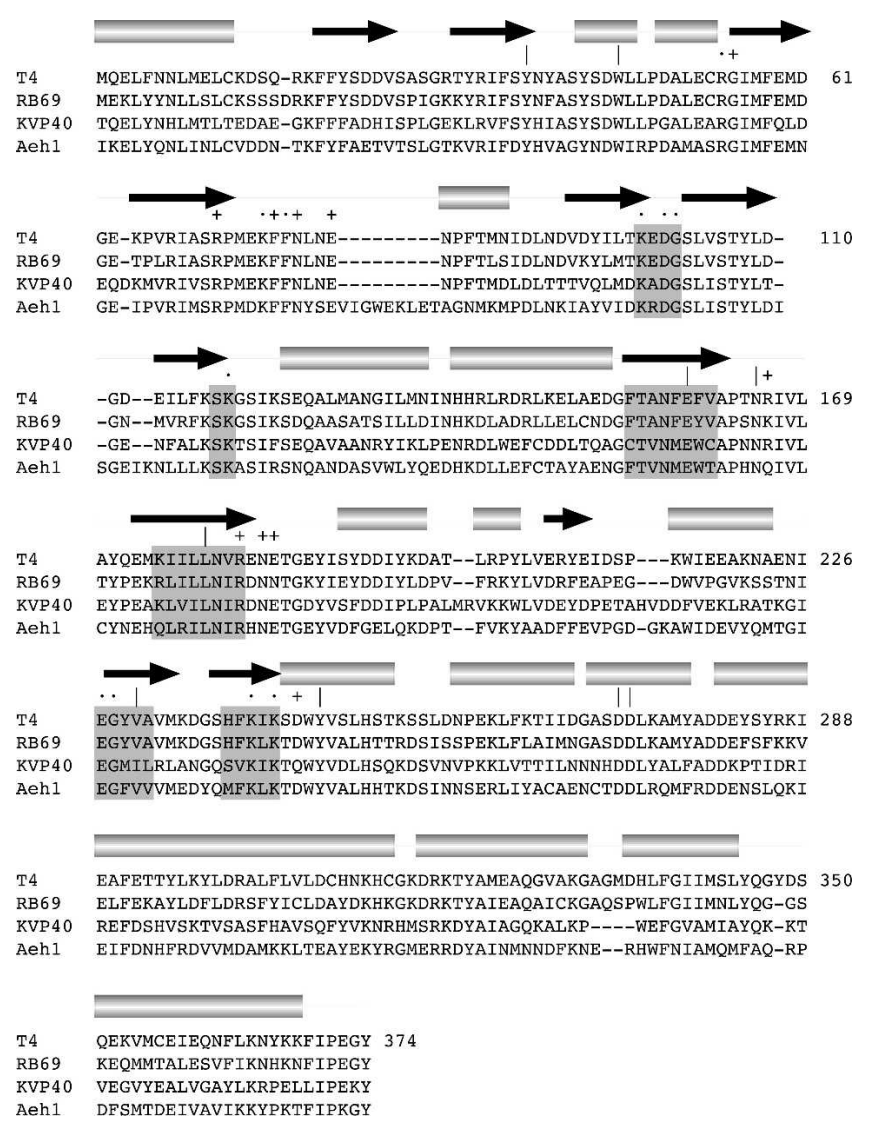

FIGURE 1. Bacteriophage RNA ligase 1. The amino acid sequence of T4 Rnl1 is aligned to the sequences of the homologous proteins of coliphage RB69, vibriophage KVP40, and Aeromonas hydrophila phage Aeh1. The secondary structure elements of T4 Rnl1 are displayed above the sequence, with $\beta$-strands indicated by arrows and helices as cylinders. Nucleotidyl transferase motifs I, Ia, III, IIIa, IV, and V are highlighted in shaded boxes. T4 Rnll residues identified previously as essential are indicated by $\mathbf{0}$. Nonessential residues are indicated by + . The nine amino acids mutated in the present study are denoted by "|."

Nandakumar and Shuman 2004; 2005) and a suite of crystal structures including (1) the N-terminal adenylyltransferase domain with noncovalently bound AMP (Ho et al. 2004); (2) the full-length Rnl2 with covalently bound AMP (Nandakumar et al. 2006); and (3) Rnl2 bound to a nicked duplex substrate (Nandakumar et al. 2006). The structure of the adenylyltransferase domain of trypanosome REL1 has been reported (Deng et al. 2004), and it is very similar to T4 Rnl2.

The basis for catalysis by the Rnl1 ligase family has begun to emerge from a combination of mutational studies (Heaphy et al. 1987; Wang et al. 2003; Wang and Shuman 2005) and a newly reported structure of T4 Rnl1 (Fig. 2A; El Omari et al. 2006). Gait and colleagues had shown 20 years ago that T4 Rnll forms a covalent enzyme-AMP intermediate at Lys99 and that mutation of Lys99 (or vicinal residue Asp101) abolishes ligase activity (Thogerson et al. 1985; Heaphy et al. 1987). Wang et al. (2003) later tested the effects of alanine mutations at 19 conserved positions in Rnll and thereby identified nine other residues essential for ligase activity. Seven essential residues are located within counterparts of conserved nucleotidyl transferase motifs I $\left({ }^{99} \underline{K E D G}{ }^{102}\right)$, Ia $\left({ }^{118} \underline{S K}^{119}\right)$, IV $\left({ }^{227}{ }^{E G Y V A}{ }^{231}\right)$, and $\mathrm{V}\left({ }^{238} \mathrm{HFKIK}^{242}\right)$ that comprise the active sites of DNA ligases and mRNA capping enzymes (Fig. 1; Wang et al. 2003; Shuman and Lima 2004). Three other essential residues, Arg54, Lys75, and Phe77, are located upstream of the AMP attachment site within a conserved domain unique to phage Rnll and yeast tRNA ligase (colored blue in Fig. 2A). We therefore proposed a shared evolutionary history and active site architecture in T4 Rnl1 (a tRNA repair enzyme) and Trl1 (a tRNA splicing enzyme).

We determined structure-activity relationships via conservative substitutions at essential residues and examined mutational effects on the isolated steps of Rnl1 adenylylation (step 1) and phosphodiester bond formation (step 3). Lys75, Lys240, and Lys242 were found to be essential for step 1 and overall ligation of $5^{\prime}$-phosphorylated RNA, but not for phosphodiester bond formation. These results suggested that the composition of the Rnll active site is different during steps 1 and 3. Of note, mutations at Arg54 and Lys119 abolished the overall RNA ligation reaction without affecting steps 1 and 3. Arg54 and Lys119 were thereby implicated as specific catalysts of the RNA adenylation reaction (step 2) of the ligation pathway (Wang et al. 2003).

El Omari et al. (2006) recently reported a $2.2 \AA$ structure of T4 Rnll that revealed a unique all-helical C-terminal domain with no counterpart in DNA ligases, capping enzymes, or Rnl2-type RNA ligases (Fig. 2A). A remarkable feature of the Rnll structure, captured with the ATP analog AMPCPP, is that the substrate adenylate moiety and many of the side chains at the active site are almost perfectly superimposable on the adenylate and active site residues of T4 Rnl2 (Ho et al. 2004; El Omari et al. 2006; Nandakumar et al. 2006). The identity of many of the active site constituents seen in the Rnll crystal structure (Fig. 2B), and even several specific atomic contacts, had been surmised based on mutational analyses (Wang et al. 2003). While nicely accounting for the available structure-function data, the Rnl1 structure also revealed candidate active site residues that were not anticipated from sequence comparisons, including side chains coordinating two metal ions (Glu159, Tyr246, Asp272, Asp273) or contacting the adenine ring of AMPCPP (Leu179, Val230), the 2'-OH (Glu159), and the $\gamma$ phosphate (Tyr37) (Fig. 2B). Glu159 and Leu179 are located within previously unrecognized equivalents of nucleotidyl transferase motifs III and IIIa (Fig. 1).

Here, guided by the T4 Rnll structure, we performed an alanine scan of the seven residues cited above and two other amino acids (Trp45, Asn165) conserved among bacteriophage Rnll homologs (the targeted residues are indicated by "|" in Fig. 1). We thereby identified Glu159 and Tyr246 
A

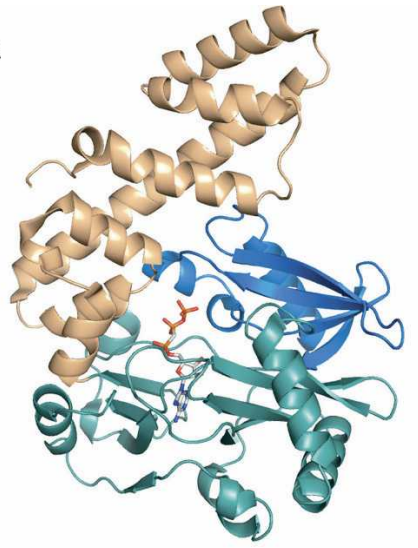

B

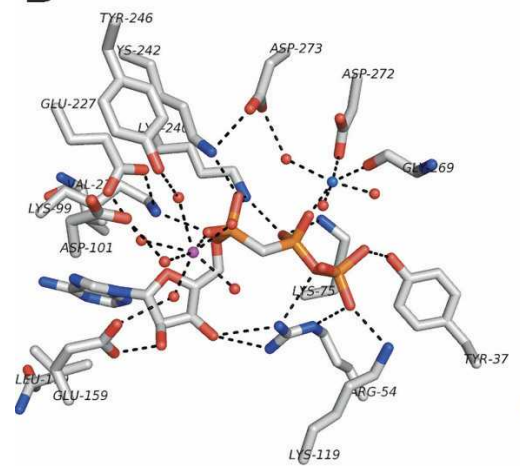

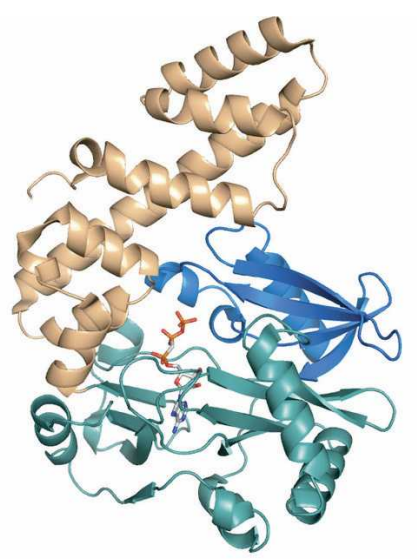

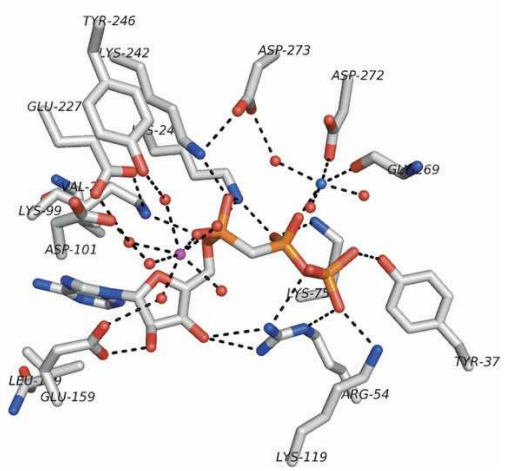

FIGURE 2. Tertiary structure and active site of T4 Rnll. (A) A stereo view of the Rnll fold (from PDB 2C5U) is shown with AMPCPP in the active site. The adenylyltransferase domain is colored green; the Rnll-specific N-terminal module is colored blue; the unique all-helical C-terminal domain is colored beige. (B) A stereo view of the Rnll active site. The calcium ion at the $\alpha$ phosphate of AMPCPP is colored magenta; the magnesium ion at the $\beta$ phosphate is colored cyan; waters are depicted as red spheres. Hydrogen bonding and ionic interactions are depicted as dashed lines. The image was prepared with Pymol.

as essential for overall ligase activity. We proceeded to delineate structure-activity relationships at Glu159, and Tyr246 and to test the present set of Rnl1 mutants for their ability to catalyze tRNA repair in vivo.

\section{RESULTS}

\section{Structure-guided alanine scan of T4 Rnl1}

Wild-type Rnl1 and mutants Y37A, W45A, E159A, N165A, L179A, V230A, Y246A, D272A, and D273A were produced in bacteria and then purified from soluble lysates by $\mathrm{Ni}-$ agarose chromatography (Fig. 3A). The proteins were assayed for RNA ligation under conditions of enzyme excess in order to illuminate the most severe catalytic defects (Fig. 3B). Reaction of wild-type Rnll with a $5^{\prime}{ }^{32} \mathrm{P}$ labeled 18-mer RNA oligonucleotide (pRNA) in the presence of $20 \mu \mathrm{M}$ ATP resulted in nearly complete conversion of the substrate to a new radiolabeled product, migrating faster than the input 18-mer pRNA strand, which corresponds to a covalently closed 18-mer circle formed by intramolecular ligation of the $5^{\prime}-\mathrm{PO}_{4}$ and $3^{\prime}-\mathrm{OH}$ termini of the RNA strand. A minor product, migrating $\sim 1$ nucleotide (nt) step slower than the input 18mer, corresponds to the RNA-adenylate (AppRNA) generated by AMP transfer from Rnl1-AMP to the $5^{\prime}$ end of the input 18-mer RNA. Mutants Y37A, D272A, and D273A also catalyzed circularization of the substrate, implying that Tyr37, Asp272, and Asp272 are nonessential for ligase function. In contrast, E159A failed to form ligated circular RNA or the RNA-adenylate intermediate. Y246A formed no circular product and only low levels of RNAadenylate. We surmise that Glu159 and Tyr246 are essential for RNA ligase activity. Four other mutants (W45A, N165A, L179A, and V230A) generated substantial circular RNA product, albeit less than wild-type Rnl1, while also forming higher levels of residual RNA-adenylate than wild-type Rnl1, suggesting a modest defect in strand sealing for these mutants.

The mutational effects on the adenylyltransferase activity of recombinant Rnl1 (step 1 of the ligation pathway) were gauged by label transfer from $[\alpha-$ $\left.{ }^{32} \mathrm{P}\right] \mathrm{ATP}$ to the Rnll polypeptide to form a covalent enzyme-adenylate adduct. The yield of Rnl1-AMP by mutants Y37A, N165A, L179A, V230A, D272A, and D273A was similar to that of wild-type Rnl1 (Fig. 3C), which is in keeping with the observation that each of these mutants was active in the composite pRNA ligation reaction. An instructive finding was that Y246A retained wild-type adenylyltransferase activity despite being grossly defective in pRNA ligation, implying that Tyr246 is critical for a downstream step in the reaction pathway. W45A and E159A yielded about half and one-fifth as much ligaseAMP, respectively, as did wild-type Rnll. Thus, a step 1 defect might contribute to, but does not entirely explain, the complete loss of overall function of the E159A protein.

\section{Mutational effects on the kinetics of single-turnover pRNA ligation}

A finer analysis of the pRNA ligation reaction by those proteins that retained activity in the single point assay (Fig. $3 \mathrm{~B}$ ) was performed by following the rate of product formation under conditions of enzyme excess (Fig. 4). RNA circularization by wild-type Rnll was complete within $5 \mathrm{~min}$, and there was little RNA-adenylate intermediate 

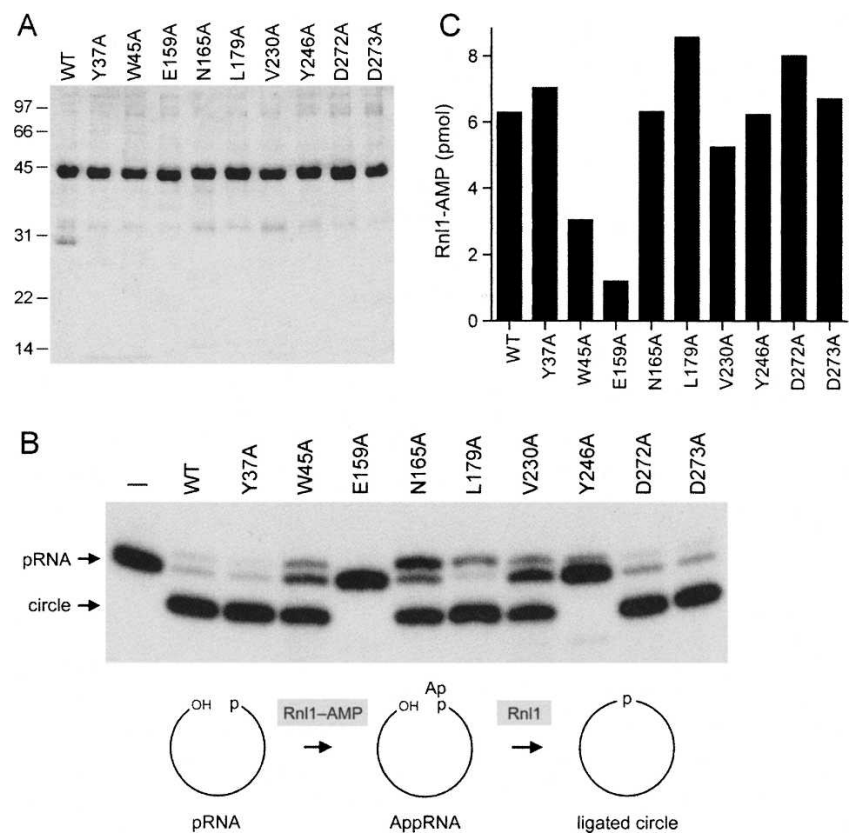

FIGURE 3. Rnl1-Ala mutants. (A) Aliquots $(5 \mu \mathrm{g})$ of recombinant wild-type (WT) and mutated Rnll proteins were analyzed by SDS-PAGE. The Coomassie blue-stained gel is shown. The positions and sizes $(\mathrm{kDa})$ of marker polypeptides are indicated on the left. (B) RNA ligation reaction mixtures $(10 \mu \mathrm{L})$ containing $50 \mathrm{mM}$ Tris- $\mathrm{HCl}(\mathrm{pH} 8.0), 10 \mathrm{mM}$ $\mathrm{MgCl}_{2}, 2 \mathrm{mM}$ DTT, $20 \mu \mathrm{M}$ ATP, 1 pmol of pRNA substrate, and $400 \mathrm{ng}$ of wild-type or mutant Rnll were incubated at $37^{\circ} \mathrm{C}$ for $30 \mathrm{~min}$. The products were resolved by PAGE and visualized by autoradiography. A control reaction lacking Rnll is shown in lane "-." The step 2 (RNA adenylation) and step 3 (phosphodiester bond formation) reactions of the ligation pathway are illustrated schematically at the bottom. (C) Adenylyltransferase specific activity was determined by label transfer from $\left[\alpha^{32} \mathrm{P}\right]$ ATP to Rnll as described in Materials and Methods.

detected at earlier times when $25 \%-50 \%$ of the substrate had been consumed (Fig. 4). Y37A, D272A, and D273A reacted with similar kinetics and did not accumulate RNAadenylate intermediate. From the data in Figure 4, we estimated that the initial rates of sealing by D272A and D273A were one-half of the wild-type value. L179A catalyzed circularization at half the initial rate of wild-type Rnl1 but attained a slightly lower endpoint at 10 min owing to the accumulation of residual RNA-adenylate (Fig. 4). Mutants V230A, N165A, and W45A were more clearly compromised, with initial rates of circularization being onetenth of the wild-type value (Fig. 4). V230A and N165A consumed nearly all of the input substrate after $30 \mathrm{~min}$, with higher than wild-type levels of residual RNA-adenylate. W45A did not consume all of the substrate in this experiment, even after $30 \mathrm{~min}$.

\section{Phosphodiester formation at a preadenylated RNA 5' end}

A preadenylated 18-mer RNA substrate (AppRNA) was used for analysis of step 3 of the ligation pathway in isolation.
Reaction of Rnll with AppRNA in enzyme excess in the absence of ATP resulted in the appearance of a sealed circular RNA product (Fig. 5). As noted previously (Wang et al. 2003), essentially no $5^{\prime}-\mathrm{PO}_{4}$ RNA strand (pRNA) was formed, signifying that the reaction was biased toward ligation (step 3) and away from deadenylation (the reverse of step 2 of the ligation pathway). Mutants Y37A, D272A, and D273A displayed wild-type extents of step 3 activity, consistent with their vigorous overall sealing function. E159A and Y246A were virtually inert in phosphodiester formation, implying that Glu159 and Tyr246 are essential for both steps 2 and 3 of the ligation pathway. N165A was able to circularize RNA-adenylate, albeit to a lesser extent that wild-type Rnl1 (Fig. 5), which might explain the presence of residual RNA-adenylate during the reaction of N165A with a pRNA substrate (Fig. 4).

The W45A, L179A, and V230A enzymes were grossly defective in the isolated step 3 reaction, while retaining substantial activity in ligase adenylation and overall pRNA ligation. These paradoxical effects, which echo those reported

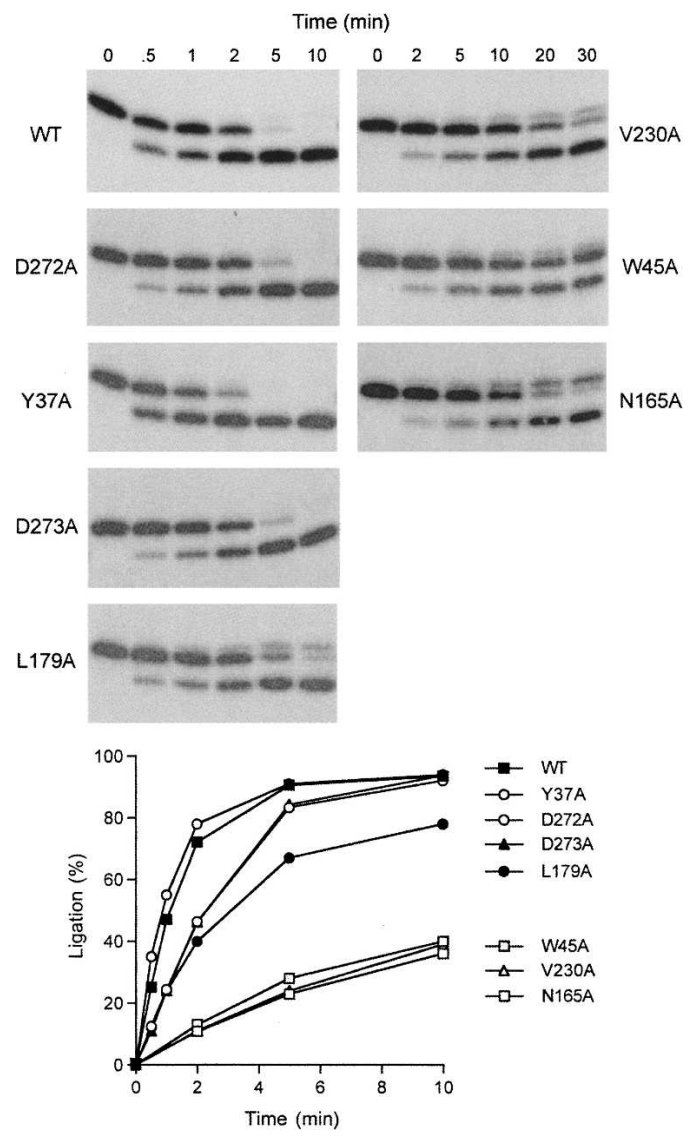

FIGURE 4. Kinetics of RNA sealing by wild-type and mutant Rnll proteins. Ligation reaction mixtures containing labeled pRNA and Rnll as specified were incubated at $37^{\circ} \mathrm{C}$. Aliquots were withdrawn and quenched at the times specified over the lanes. The products were resolved by PAGE and visualized by autoradiography. The yield of circular RNA product is plotted as a function of time at bottom. 


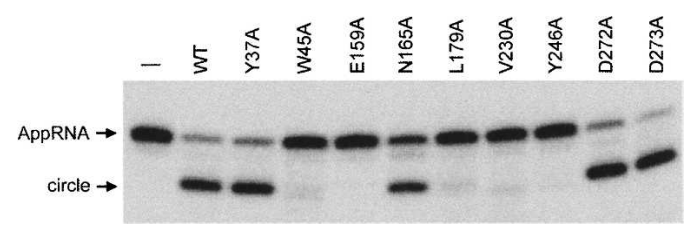

FIGURE 5. Effects of alanine mutations on ligation of a preadenylated RNA. Reactions mixtures contained radiolabeled AppRNA and Rnll as specified. The products were resolved by PAGE and visualized by autoradiography. Rnll was omitted from a control reaction (lane "-").

previously for Arg182 mutations (Wang et al. 2003), indicate that the sealing of an exogenous preformed AppRNA differs in certain respects from the sealing of the AppRNA intermediate formed in situ on the enzyme.

\section{Structure-activity relationships at Glu159 and Tyr246}

To further evaluate the contributions of the two newly defined essential residues (Glu159 and Tyr246) to the RNA ligase reaction, we tested the effects of conservative substitutions. Glu159 was replaced by glutamine and aspartate; Tyr246 was changed to phenylalanine, leucine, and serine. The conservative mutants were produced in Escherichia coli and purified from soluble bacterial extracts by Ni-agarose chromatography; SDS-PAGE (data not shown) confirmed that their purity was similar to that seen in Figure 3A for Rnl1-Ala mutants. Single point assays of pRNA sealing by excess Rnl1 revealed that E159Q was unreactive, highlighting the requirement for a carboxylate functional group (Fig. 6A). E159D was also defective, catalyzing scant conversion of pRNA to circular product and RNA-adenylate intermediate under conditions in which wild-type Rnl1 circularized all of the substrate. We surmise that the distance from the main chain to the carboxylate is critical for the function of this residue. The finding that pRNA sealing was abolished by the leucine and serine changes at Tyr246 indicates that a hydroxyl is not sufficient and an aromatic moiety is essential (Fig. 6A). The latter point is underscored by the partial restoration of function by introducing phenylalanine. The initial rate of circularization by $\mathrm{Y} 246 \mathrm{~F}$ was $4 \%$ of the wild-type value (Fig. 6B).

\section{Mutational effects on tRNA repair activity in vivo}

The enzymatic steps in bacteriophage tRNA repair are broadly similar to those of yeast tRNA splicing. The endhealing and strand-sealing steps of the phage T4-encoded tRNA repair pathway are performed by Pnkp and Rnll, whereas a single polypeptide Trl1 (composed of discrete healing and sealing domains) performs these steps during yeast tRNA splicing (Amitsur et al. 1987; Sawaya et al. 2003). We have shown that RNA repair systems are portable in vivo, insofar as a lethal trl1 $\Delta$ mutation of Saccharomyces cerevisiae can be rescued to normal growth by coexpression of bac- teriophage T4 Rnl1 and Pnkp (Schwer et al. 2004). This approach provides a means to test structure-function relationships for RNA repair enzymes by functional complementation in yeast.

A plasmid-shuffle complementation assay revealed that coexpression of wild-type T4 Rnl1 and T4 Pnkp (in a CEN plasmid under the control of the yeast SLU7 promoter) rescued the $\operatorname{trl} 1 \Delta$ mutation at $18^{\circ} \mathrm{C}, 30^{\circ} \mathrm{C}$, and $37^{\circ} \mathrm{C}$ (Table $1)$. We found that whereas the six Rnl1 mutants that were defective for pRNA ligation in vitro (E159A, E159D, E159Q, Y246A, Y246S, Y246L) were unable to support tRNA splicing in vivo at any temperature tested, the Rnl1-Ala mutants that displayed wild-type or near wild-type pRNA sealing activity in vitro (Y37A, D272A, D273A, L179A) were active in vivo in yeast (Table 1). Even the Rnll mutants with reduced sealing activity in vitro (W45A, N165A, V230A, Y246F) were capable of complementing the trlls strain (Table 1).

\section{DISCUSSION}

T4 Rnl1 is a bona fide RNA repair enzyme that plays a critical role in thwarting the tRNA-damaging antiviral response of the host bacterium. Although the reaction scheme has been known for many years (Uhlenbeck and Gumport 1982), the structural basis for catalysis has only recently been appreciated as mutational and crystallographic approaches have converged. Rnll consists of three modules: (1) a nucleotidyl transferase domain (amino acids 71-242) (colored green in Fig. 2A) composed of a cage of $\beta$ strands that form a nucleotide-binding pocket; (2) a
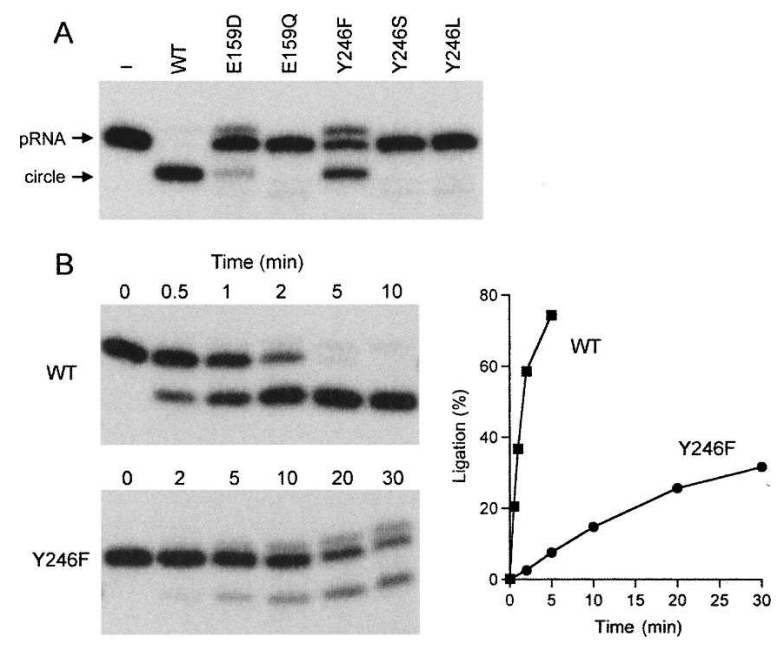

FIGURE 6. Effects of conservative mutations of Glu159 and Tyr246. (A) Reaction mixtures $(10 \mu \mathrm{L})$ containing $50 \mathrm{mM}$ Tris- $\mathrm{HCl}(\mathrm{pH} 8.0)$, $10 \mathrm{mM} \mathrm{MgCl}_{2}, 2 \mathrm{mM}$ DTT, $20 \mu \mathrm{M}$ ATP, 1 pmol of pRNA substrate, and $400 \mathrm{ng}$ of wild-type or mutant Rnl1 were incubated at $37^{\circ} \mathrm{C}$ for $30 \mathrm{~min}$. A control reaction lacking Rnl1 is shown in lane "-." (B) Kinetic analysis of single turnover pRNA ligation was performed as described in Materials and Methods. The product analysis is shown in the left panel; the extent of circularization is plotted as a function of time in the right panel. 
TABLE 1. Mutational effects on Rnl1 tRNA repair activity in vivo

\begin{tabular}{lccc}
\hline & \multicolumn{3}{c}{ trl1 $\Delta$ complementation } \\
\cline { 2 - 4 } Rn11 & $18^{\circ} \mathrm{C}$ & $30^{\circ} \mathrm{C}$ & $37^{\circ} \mathrm{C}$ \\
\hline Wild type & +++ & +++ & +++ \\
Y37A & +++ & +++ & +++ \\
W45A & +++ & +++ & ++ \\
E159A & - & - & - \\
E159D & - & - & - \\
E159Q & - & - & - \\
N165A & +++ & +++ & ++ \\
L179A & +++ & +++ & +++ \\
V230A & +++ & +++ & ++ \\
Y246A & - & - & - \\
Y246F & +++ & +++ & +++ \\
Y246L & - & - & - \\
Y246S & - & - & - \\
D272A & ++ & +++ & ++ \\
D273A & ++ & +++ & +++ \\
\hline
\end{tabular}

70-amino acid N-terminal module specific to the Rnl1 subfamily (colored blue in Fig. 2A); and (3) an all-helical C-terminal domain (amino acids 243-372, colored beige) that is unrelated to the $\mathrm{C}$-terminal domains of DNA ligases or T4 Rnl2 (El Omari et al. 2006).

The structure of Rnll bound to the unreactive analog AMPCPP and two metal ions plausibly mimics the Michaelis complex immediately prior to catalysis of the ligase adenylylation reaction. The Rnl1 Lys99 is apical to the pyrophosphate leaving group, with $\mathrm{N} \zeta$ located $3.5 \AA$ from the $\alpha$ phosphorus and in a suitable orientation for in-line attack. The constellation of previously identified essential residues that comprise the Rn1 active site includes three that contact the $\alpha$ phosphate of AMPCPP (Lys99, Lys240, Lys242) and four that contact the $\beta$ or $\gamma$ phosphates (Arg54, Lys75, Lys119, Lys240) (Fig. 2B). Here we found that elimination of Tyr37, which contacts the $\gamma$ phosphate in the crystal structure, has no discernible impact on Rnl1 activity in vitro or in vivo. This is a remarkable finding given that Tyr37 is the sole protein contact to the $\mathrm{O} 3 \gamma$ atom and is conserved as a tyrosine in other phage Rnll homologs (Fig. 1).

Two divalent metals are seen in the Rnll active site: a magnesium ion that interacts with the $\beta$ phosphate and a calcium ion that coordinates the $\alpha$ phosphate (Fig. 2B). Ala mutations of the two magnesium-binding residues (Asp272 and Asp273) had no significant impact on Rnl1 activity. The Asp-Asp pair is conserved in other phage Rnll proteins. Asp272 coordinates the magnesium directly, whereas Asp273 interacts indirectly via a water of the octahedral magnesium complex. Asp273 also forms an ion pair with essential motif $V$ residue Lys242 (Fig. 2B). Apparently neither contact of Asp273 is essential. Although a two-metal mechanism for the ligase adenylylation reaction has been discussed (El Omari et al. 2006), the present data raise the possibility that proper orientation of the leaving group in step 1 is attainable via the extensive network of phosphate contacts with lysine and arginine side chains, either in the absence of the magnesium seen in the ligase structure or with an alternative magnesium coordination complex in which the D272 carboxylate atom is replaced by water.

In contrast, the side-chain components of the calcium coordination complex to the $\alpha$ phosphate are all essential for Rnl1 activity. These include Asp101 (motif I), Glu227 (motif IV), and the two residues identified here as critical for catalysis: Tyr246 and Glu159. Calcium is not a likely physiological cofactor for Rnl1; the "correct" cofactor is presumed to be magnesium. The calcium in the Rnll structure reflects the use of high concentrations of this metal in the crystallization buffer (El Omari et al. 2006). Yet, the location of the calcium in the Rnl1 step 1 substrate complex is close to that of magnesium in the covalent Rnl2-AMP intermediate (the step 1 product complex) (Nandakumar et al. 2006), suggesting that the calcium site in Rnll is the correct one occupied by magnesium for catalysis of ligaseadenylylation. It is notable that the calcium coordinates seven oxygen atoms (six from water and one from the $\alpha$ phosphate). It would be expected that magnesium forms an octahedral complex with six constituents.

The essential Tyr246 residue is located within the first helix of the unique C-terminal domain of Rnl1 and is conserved in other phage Rnl1 enzymes. The crystal structure suggests that the Y246 OH receives a hydrogen bond from a water in the calcium coordination complex and donates a hydrogen bond to the Asp101 carboxylate. Yet the contacts seen in the step 1 substrate complex are not likely to reflect the key roles of Tyr246 and Asp101, insofar as Asp101 was shown by the Gait laboratory to be dispensable for ligaseAMP formation (Heaphy et al. 1987) and we report here that replacing Tyr246 with alanine had no effect on the extent of ligase adenylylation in vitro. Our findings point to essential functions of Tyr246 during steps 2 and 3 of the ligation pathway (similar to the requirement for Asp101 in steps 2 and 3 noted previously). The structure-activity relationships at Tyr246 indicate that hydrogen bonding via the hydroxyl is not the sole critical contribution of this residue. The Y246F change restores ligase activity partially compared with Y246A (to 4\% of the wild-type rate in vitro), and this level of activity appears to suffice for tRNA splicing in yeast. The finding that the Y246L mutant is inactive in vitro and in vivo suggests that the aromatic quality of the side chain, or the $\mathrm{C} \varepsilon$ and $\mathrm{Cz}$ atoms of the phenol ring, is essential. Indeed, the phenol ring of Tyr246 stacks closely on the essential Glu227 side chain, making multiple van der Waals contacts, as follows: (1) Tyr C $\delta 1$ to Glu C $\alpha$ (3.6 ̊);

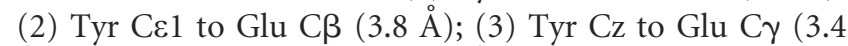
$\AA)$; and Tyr O to Glu C $\delta(3.7 \AA)$. We surmise that these stacking interactions, together with the hydrogen bond contacts cited above, comprise the key contributions of Tyr246 to catalysis of steps 2 and 3. Because Tyr246 is situated 
above the AMP moiety on the enzyme surface, it is also possible that it makes contact with one of the RNA termini during the steps of RNA adenylylation and phosphodiester formation.

Glu159 is essential for Rnl1 activity in vitro and in vivo. In addition to its water-mediated contact to the calcium ion, Glu159 accepts a hydrogen bond from the ribose O2' of AMPCPP (Fig. 2B). Glu159 is located within the Rnl1 counterpart of nucleotidyltransferase motif III (FTANFEFV $^{161}$ ) (Fig. 1) found in all branches of the ligase/capping enzyme superfamily. The motif III glutamate contacts the ribose sugar of the NTP substrate, the covalent enzymeNMP intermediate, or the polynucleotide-adenylate intermediate in various crystal structures of nucleotidyltransferase superfamily members (Subramanya et al. 1996; Håkansson et al. 1997; Pascal et al. 2004; Nandakumar et al. 2006). The original consensus motif III element was defined as $\varphi \varphi \varphi$ DGE $\varphi \varphi$ (where $\varphi$ is an aliphatic side chain). Later studies showed that the aspartate preceding the glycine, although essential when present in ligases or capping enzymes, is not strictly conserved in all nucleotidyltransferases (Wang et al. 1997). Also, the glycine is replaced in some ligases and capping enzymes by alanine, serine, cysteine, or threonine. The glutamate is the only strictly conserved motif III residue, and it is essential for ligase or capping enzyme activity in all cases tested (Sriskanda and Shuman 2002; Sawaya and Shuman 2003; Yin et al. 2003; Zhu and Shuman 2005). We find that Glu159 is strictly essential in Rnll, because conservative substitutions with glutamine or aspartate abolished or suppressed pRNA sealing and were lethal in vivo. These findings clarify that Rnl1 uses the same complement of essential motifs within the nucleotidyltransferase domain as do other members of the superfamily. Glu159 is one of two essential side chains that contact the adenylate ribose, the other being Arg54, which coordinates the O3' atom (Fig. 2B).

Rnl1, like Rnl2, makes no side-chain contacts to the edge of the adenine base. The adenine occupies a hydrophobic pocket, where it is sandwiched between residues Leu179 (motif IIIa) and Val230 (motif IV). The adenine sandwich in Rnl1 is a variant of the aromatic(IIIa)-purine-hydrophobic (IV) stack characteristic of other polynucleotide ligases and RNA capping enzymes. Changing Leu179 and Val230 to alanine had little effect on the extent of Rnl1 adenylylation in vitro and resulted in rate decrements of twofold and 10-fold, respectively, for single-turnover pRNA ligation. Nonetheless, the L179A and V230A mutants were able to support tRNA splicing when expressed in yeast. Paradoxically, the L179A and V230A mutations abolished the sealing of preformed AppRNA by Rnl1 but spared the overall pRNA ligation reaction, which perforce requires catalysis of step 3. The explanation offered previously for this anomaly was that sealing of an exogenous AppRNA substrate entails a step in the binding of free AppDNA that does not apply when the adenylated polynucleotide in- termediate is formed in situ at the ligase active site (Wang et al. 2003). We infer that the adenine sandwich is more stringently required for binding the $5^{\prime}$ adenylate of free AppRNA than it is for binding ATP. We also infer a role for the sandwich in retaining the AppRNA intermediate formed during the composite pRNA ligation reaction, insofar as the L179A and V230A mutants accumulate higher than normal levels of residual RNA adenylate. As discussed extensively for DNA ligase and Rnl2 (Sriskanda and Shuman 1998; Ho and Shuman 2002), the trapping of residual polynucleotide adenylate reflects premature release of the step 2 product and subsequent reaction of the ligase apoenzyme with ATP to form ligase-AMP, which can no longer react with free AppRNA. We had seen previously that mutations of Arg182 in Rnl1 also selectively block the sealing of exogenous AppRNA but have little effect when the RNAadenylate is formed in situ. The Rnl1 crystal structure clarifies that Arg182, which is part of the motif IIIa $\beta$ strand (Fig. 1), forms a bidentate salt bridge to Glu52 on the back side of the enzyme away from the active site; we suspect that this ion pair stabilizes the interface of the adenylyltransferase domain with the Rnl1-specific N-terminal module.

Alanine mutations at two other conserved Rnl1 residues_-Trp45 and Asn165_-also result in slowing of singleturnover pRNA ligation, albeit not severe enough to affect tRNA splicing in yeast. Neither Trp45 not Asn165 is a constituent of the active site of the Rnl1-AMPCPP substrate complex. N165A displays a partial defect in the isolated step 3 reaction and accumulates AppRNA during a reaction with pRNA, suggesting an effect on RNA binding. Asn165 is located on the surface of the adenylyltransferase domain near the rim of the adenylate pocket; the Asn $\mathrm{O} \delta 1$ accepts a hydrogen bond from the hydroxyl of Ser103 (flanking motif I). It is conceivable that Asn 165 stabilizes the active conformation of the motif I interstrand loop (Fig. 1); alternatively, Asn 165 might interact directly with one of the ends of the RNA substrate.

Changing Trp45 to alanine abolished sealing of preformed AppRNA. While not itself a constituent of the active site, Trp45 residues within a hydrophobic core of the protein at the interface of the adenylyltransferase domain and N-terminal module. Trp45 makes van der Waals contacts to Tyr37 (which is part of the active site, although not essential) and to Phe77. We showed previously that changing Phe77 to alanine severely inhibited overall pRNA ligation, ligaseadenylylation, and the sealing of preformed AppRNA (Wang et al. 2003). We surmise that the hydrophobic core to which Trp45 and Phe77 contribute is important for the proper folding of Rnl1.

In summary, we have exploited the recent structure of Rnl1 (El Omari et al. 2006) to complete a functional dissection of the active site. Our findings underscore the theme that crystallographic contacts are not always predictive of stringent functional relevance. The constellation of Rnl1 side-chains essential for activity now includes 11 residues: 
Arg54, Lys75, Phe77, Lys99, Asp101, Lys119, Glu159, Glu227, Lys240, Lys242, and Tyr246. The major outstanding questions concerning Rnll now pertain to the nature of the RNA-binding surface and the issue of whether Rnll specifically recognizes broken tRNAs.

\section{MATERIALS AND METHODS}

\section{Recombinant T4 Rnl1}

Amino acid substitution mutations were introduced into the rnll gene by PCR using the two-stage overlap extension method as described previously (Wang et al. 2003). The mutated genes were digested with NdeI and BamHI and then inserted into pET16b. The plasmid inserts were sequenced completely to exclude the acquisition of unwanted changes during amplification and cloning. Wildtype and mutant pET-RNL1 plasmids were transformed into E. coli BL21(DE3). Induction of Rnl1 production with IPTG and purification of $\mathrm{His}_{10}$-Rnll from soluble bacterial extracts by Ni-agarose chromatography were performed as described previously (Wang et al. 2003). Protein concentrations were determined with the BioRad dye reagent using bovine serum albumin as the standard.

\section{RNA ligase assay}

An 18-mer oligoribonucleotide (5'-AUUCCGAUAGUGACUACA) was $5^{\prime}{ }^{32} \mathrm{P}$-labeled using T4 polynucleotide kinase and $\left[\gamma^{-}{ }^{32} \mathrm{P}\right] \mathrm{ATP}$. The labeled 18-mer was purified by electrophoresis through a $20 \%$ polyacrylamide gel. RNA ligation reaction mixtures $(10 \mu \mathrm{L})$ containing $50 \mathrm{mM}$ Tris- $\mathrm{HCl}$ (pH 8.0), $2 \mathrm{mM} \mathrm{DTT}, 10 \mathrm{mM} \mathrm{MgCl}_{2}$, 1 pmol of $5^{\prime}{ }^{32} \mathrm{P}$-labeled 18-mer RNA (pRNA), $20 \mu \mathrm{M}$ ATP, and $400 \mathrm{ng} \mathrm{Rnll}$ were incubated for $30 \mathrm{~min}$ at $37^{\circ} \mathrm{C}$. The reactions were quenched by adding $5 \mu \mathrm{L}$ of $95 \%$ formamide and $20 \mathrm{mM}$ EDTA. For kinetic analysis of RNA sealing, reaction mixtures $(80 \mu \mathrm{L})$ containing $50 \mathrm{mM}$ Tris- $\mathrm{HCl}(\mathrm{pH} 8.0), 2 \mathrm{mM}$ DTT, $10 \mathrm{mM} \mathrm{MgCl}_{2}, 8 \mathrm{pmol}$ of labeled pRNA, $20 \mu \mathrm{M}$ ATP, and $3.2 \mu \mathrm{g}$ $\mathrm{Rnll}$ were incubated at $37^{\circ} \mathrm{C}$. The reactions were initiated by adding enzyme. Aliquots $(10 \mu \mathrm{L})$ were withdrawn at the time specified and quenched immediately with formamide/EDTA. The samples were analyzed by electrophoresis through an $18 \%$ polyacrylamide gel containing $7 \mathrm{M}$ urea in $0.5 \times \mathrm{TBE}(45 \mathrm{mM}$ Tris-borate, $1 \mathrm{mM}$ EDTA). The ligation reaction products were visualized by autoradiography of the gel and quantified with a Fujifilm BAS-2500 imager.

\section{Adenylyltransferase assay}

Reaction mixtures $(20 \mu \mathrm{L})$ containing $50 \mathrm{mM}$ Tris- $\mathrm{HCl}(\mathrm{pH} 8.0), 2$ mM DTT, $5 \mathrm{mM} \mathrm{MgCl}_{2}, 20 \mu \mathrm{M}\left[\alpha_{-}{ }^{32} \mathrm{P}\right] \mathrm{ATP}$, and $0.5,1.0$, or $1.5 \mu \mathrm{g}$ of wild-type or mutant Rnll were incubated for $12 \mathrm{~min}$ at $37^{\circ} \mathrm{C}$. The reactions were quenched with SDS, and the products were analyzed by SDS-PAGE. The ligase- $\left[{ }^{32} \mathrm{P}\right] \mathrm{AMP}$ adduct was visualized by autoradiography of the dried gel and quantified by scanning the gel with a Fujifilm BAS-2500 imager. The adenylyltransferase specific activities (pmol ligase- $\left[{ }^{32} \mathrm{P}\right]$ AMP formed per microgram of protein) were calculated from the slopes of the titration curves.

\section{Sealing of preformed RNA-adenylate}

Reaction mixtures $(10 \mu \mathrm{L})$ containing $50 \mathrm{mM}$ Tris- $\mathrm{HCl}(\mathrm{pH} 8.0)$, $2 \mathrm{mM}$ DTT, $10 \mathrm{mM} \mathrm{MgCl}_{2}, 0.2$ pmol of $5^{\prime}{ }^{32} \mathrm{P}$-labeled RNA- adenylate strand AppAUUCCGAUAGUGACUACA (synthesized and gel-purified as described in Yin et al. 2003), and $400 \mathrm{ng}$ Rnll as specified were incubated for $30 \mathrm{~min}$ at $37^{\circ} \mathrm{C}$. The reactions were quenched with formamide/EDTA and the products analyzed by PAGE.

\section{Test of Rnl1 tRNA repair function by plasmid shuffle}

The $\operatorname{trl} 1 \Delta$ haploid strain YRS1 (Sawaya et al. 2003) was cotransformed with a CEN TRP1 RNL1 plasmid bearing a wild-type or mutated version of T4 Rnl1 under the control of the yeast TPI1 promoter and a CEN HIS3 PNKP plasmid expressing bacteriophage T4 Pnkp under the control of the yeast SLU7 promoter. Transformants were selected on medium lacking tryptophan and histidine. Two individual colonies were transferred to fresh selective medium. The isolates were then streaked on agar medium containing $0.75 \mathrm{mg} / \mathrm{mL} 5$-FOA. The plates were incubated at $18^{\circ} \mathrm{C}, 30^{\circ} \mathrm{C}$, and $37^{\circ} \mathrm{C}$. Lethal mutations were those that did not allow formation of FOA-resistant colonies after 7-10 d at any of the temperatures tested (scored as "-" in Table 1). Other mutated alleles supported FOA-resistant colony formation at one or more of the growth temperatures. Individual colonies were picked from the FOA plate, transferred to yeast extract/peptone/dextrose (YPD) medium, and then tested for growth on YPD agar at $18^{\circ} \mathrm{C}, 30^{\circ} \mathrm{C}$, and $37^{\circ} \mathrm{C}$. RNL1 strains that formed "wild-type" sized colonies were scored as " +++ ." Strains that formed smaller colonies were scored as " ++ ."

\section{ACKNOWLEDGMENTS}

This work was supported by NIH grant GM42498. S.S. is an American Cancer Society Research Professor.

Received August 15, 2006; accepted September 18, 2006.

\section{REFERENCES}

Amitsur, M., Levitz, R., and Kaufman, G. 1987. Bacteriophage T4 anticodon nuclease, polynucleotide kinase, and RNA ligase reprocess the host lysine tRNA. EMBO J. 6: 2499-2503.

Blondal, T., Thorisdottir, A., Unnsteinsdottir, U., Hjorleifsdottir, S., Aevarsson, A., Ernstsson, S., Fridjonsson, O.H., Skirnisdottir, S., Wheat, J.O., Hermannsdottir, A.G., et al. 2005. Isolation and characterization of a thermostable RNA ligase 1 from a Thermus scotoductus bacteriophage TS2126 with good single-stranded DNA ligation properties. Nucleic Acids Res. 33: 135-142.

Cranston, J.W., Silber, R., Malathi, V.G., and Hurwitz, J. 1974. Studies on ribonucleic acid ligase: Characterization of an adenosine triphosphate-inorganic pyrophosphate exchange reaction and demonstration of an enzyme-adenylate complex with T4 bacteriophage-induced enzyme. J. Biol. Chem. 249: 7447-7456.

Deng, J., Schnaufer, A., Salavati, R., Stuart, K.D., and Hol, W.G.J. 2004. High resolution crystal structure of a key editosome enzyme from Trypanosoma brucei: RNA editing ligase 1. J. Mol. Biol. 343: 601-613.

El Omari, K., Ren, J., Bird, L.E., Bona, M.K., Klarmann, G., LeGrice, S.F.J., and Stammers, D.K. 2006. Molecular architecture and ligand recognition determinants for T4 RNA ligase. J. Biol. Chem. 281: 1573-1579.

Englert, M. and Beier, H. 2005. Plant tRNA ligases are multifunctional enzymes that have diverged in sequence and substrate specificity from RNA ligases of other phylogenetic origins. Nucleic Acids Res. 33: $388-399$. 
Håkansson, K., Doherty, A.J., Shuman, S., and Wigley, D.B. 1997. $\mathrm{X}$-ray crystallography reveals a large conformational change during guanyl transfer by mRNA capping enzymes. Cell 89: 545-553.

Heaphy, S., Singh, M., and Gait, M.J. 1987. Effects of single amino acid changes in the region of the adenylylation site of T4 RNA ligase. Biochemistry 26: 1688-1696.

Ho, C.K. and Shuman, S. 2002. Bacteriophage T4 RNA ligase 2 (gp24.1) exemplifies a family of RNA ligases found in all phylogenetic domains. Proc. Natl. Acad. Sci. 99: 12709-12714.

Ho, C.K., Wang, L.K., Lima, C.D., and Shuman, S. 2004. Structure and mechanism of RNA ligase. Structure 12: 327-339.

Martins, A. and Shuman, S. 2004. Characterization of a baculovirus enzyme with RNA ligase, polynucleotide $5^{\prime}$ kinase and polynucleotide 3' phosphatase activities. J. Biol. Chem. 279: 18220-18231.

McManus, M.T., Shimamura, M., Grams, J., and Hajduk, S.L. 2001. Identification of candidate mitochondrial RNA editing ligases from Trypanosoma brucei. RNA 7: 167-175.

Nandakumar, J. and Shuman, S. 2004. How an RNA ligase discriminates RNA damage versus DNA damage. Mol. Cell 16: 211-221.

Nandakumar, J. and Shuman, S. 2005. Dual mechanisms whereby a broken RNA end assists the catalysis of its repair by T4 RNA ligase 2. J. Biol. Chem. 280: 23484-23489.

Nandakumar, J., Ho, C.K., Lima, C.D., and Shuman, S. 2004. RNA substrate specificity and structure-guided mutational analysis of bacteriophage T4 RNA ligase 2. J. Biol. Chem. 279: 31337-31347.

Nandakumar, J., Shuman, S., and Lima, C.D. 2006. RNA ligase structures reveal the basis for RNA specificity and conformational changes that drive the reaction forward. Cell 127: 71-84.

Pascal, J.M., O’Brien, P.J., Tomkinson, A.E., and Ellenberger, T. 2004. Human DNA ligase I completely encircles and partially unwinds nicked DNA. Nature 432: 473-478.

Phizicky, E.M., Consaul, S.A., Nehrke, K.W., and Abelson, J. 1992. Yeast tRNA ligase mutants are nonviable and accumulate tRNA splicing intermediates. J. Biol. Chem. 267: 4577-4582.

Sawaya, R. and Shuman, S. 2003. Mutational analysis of the guanylyltransferase component of mammalian mRNA capping enzyme. Biochemistry 42: 8240-8249.

Sawaya, R., Schwer, B., and Shuman, S. 2003. Genetic and biochemical analysis of the functional domains of yeast tRNA ligase. J. Biol. Chem. 278: 43298-43398.

Schnaufer, A., Panigrahi, A.K., Panicucci, B., Igo, R.P., Salavati, R., and Stuart, K. 2001. An RNA ligase essential for RNA editing and survival of the bloodstream form of Trypanosoma brucei. Science 291: 2159-2162.
Schwer, B., Sawaya, R., Ho, C.K., and Shuman, S. 2004. Portability and fidelity of RNA-repair systems. Proc. Natl. Acad. Sci. 101: 2788-2793.

Shuman, S. and Lima, C.D. 2004. The polynucleotide ligase and RNA capping enzyme superfamily of covalent nucleotidyltransferases. Curr. Opin. Struct. Biol. 14: 757-764.

Silber, R., Malathi, V.G., and Hurwitz, J. 1972. Purification and properties of bacteriophage T4-induced RNA ligase. Proc. Natl. Acad. Sci. 69: 3009-3013.

Sriskanda, V. and Shuman, S. 1998. Mutational analysis of Chlorella virus DNA ligase: catalytic roles of domain I and motif VI. Nucleic Acids Res. 26: 4618-4625.

Sriskanda, V. and Shuman, S. 2002. Role of nucleotidyl transferase motifs I, III and IV in the catalysis of phosphodiester bond formation by Chlorella virus DNA ligase. Nucleic Acids Res. 30: 903-911.

Subramanya, H.S., Doherty, A.J., Ashford, S.R., and Wigley, D.B. 1996. Crystal structure of an ATP-dependent DNA ligase from bacteriophage T7. Cell 85: 607-615.

Sugino, A., Snopek, T.J., and Cozzarelli, N.R. 1977. Bacteriophage T4 RNA ligase: Reaction intermediates and interaction of substrates. J. Biol. Chem. 252: 1732-1738.

Thogersen, H.C., Morris, H.R., Rand, K.N., and Gait, M.J. 1985. Location of the adenylylation site in T4 RNA ligase. Eur. J. Biochem. 147: 325-329.

Uhlenbeck, O.C. and Gumport, R.I. 1982. T4 RNA ligase. The Enzymes 15: 31-58.

Wang, L.K. and Shuman, S. 2005. Structure-function analysis of yeast tRNA ligase. RNA 11: 966-975.

Wang, S.P., Deng, L., Ho, C.K., and Shuman, S. 1997. Phylogeny of mRNA capping enzymes. Proc. Natl. Acad. Sci. 94: 9573-9578.

Wang, L.K., Ho, C.K., Pei, Y., and Shuman, S. 2003. Mutational analysis of bacteriophage T4 RNA ligase 1: Different functional groups are required for the nucleotidyl transfer and phosphodiester bond formation steps of the ligation reaction. J. Biol. Chem. 278: 29454-29462.

Wang, L.K., Schwer, B., Englert, M., Beier, H., and Shuman, S. 2006. Structure-function analysis of the kinase-CPD domain of yeast tRNA ligase (Trl1) and requirements for complementation of tRNA splicing by a plant Trl1 homolog. Nucleic Acids Res. 34: 517-527.

Yin, S., Ho, C.K., and Shuman, S. 2003. Structure-function analysis of T4 RNA ligase 2. J. Biol. Chem. 278: 17601-17608.

Zhu, H. and Shuman, S. 2005. Structure-guided mutational analysis of the nucleotidyltransferase domain of Escherichia coli NAD+dependent DNA Ligase (LigA). J. Biol. Chem. 280: 12137-12144. 

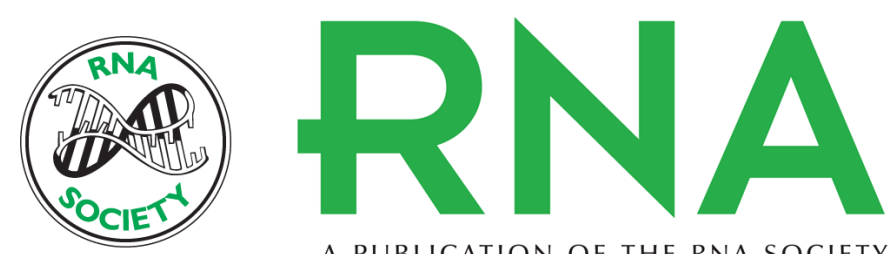

A PUBLICATION OF THE RNA SOCIETY

\section{Structure-guided mutational analysis of T4 RNA ligase 1}

Li Kai Wang, Beate Schwer and Stewart Shuman

RNA 2006 12: 2126-2134 originally published online October 26, 2006

Access the most recent version at doi:10.1261/rna.271706

$\begin{array}{ll}\text { References } & \text { This article cites } 36 \text { articles, } 17 \text { of which can be accessed free at: } \\ \text { http://rnajournal.cshlp.org/content/12/12/2126.full.htm|\#ref-list-1 }\end{array}$

License

Email Alerting Receive free email alerts when new articles cite this article - sign up in the box at the Service top right corner of the article or click here.

To subscribe to RNA go to:

http://rnajournal.cshlp.org/subscriptions 